\title{
EPIDEMIOLOGÍA, RESULTADOS CLÍNICOS Y COSTOS DEL TRATAMIENTO DEL ABSCESO TUBOOVÁRICO, EN UN HOSPITAL PÚBLICO DE SANTIAGO
}

\author{
Alfredo Ovalle S. ${ }^{1}$, Andrés Casanova B. ${ }^{1}$, Elena Kakarieka W. ${ }^{2}$, \\ Francisca de Jourdan H. ${ }^{1}$, Karin Salgado M. ${ }^{1}$
}

\footnotetext{
1 Servicio y Departamento de Obstetricia, Ginecología y Neonatología, Hospital Clínico San Borja Arriarán, Facultad de Medicina, Universidad de Chile. ${ }^{2}$ Servicio de Anatomía Patológica, Hospital Clínico, San Borja Arriarán.
}

\section{RESUMEN}

Objetivos: Conocer frecuencia y factores de riesgo del absceso tuboovárico (ATO), complicaciones y costos del tratamiento. Método: Estudio retrospectivo de 64 pacientes operadas con el diagnóstico de ATO. Las pacientes se manejaron con criterio médico-quirúrgico: uso de antibióticos y cirugía en casos de peritonitis difusa, fiebre persistente con masa palpable y masa anexial mayor de $6 \mathrm{~cm}$ sin fiebre. Se evaluaron en fertilidad futura y en complicaciones: infección y dehiscencia de herida operatoria, reoperación por ATO residual, lesión intestinal, lesión vesical y complicaciones médicas. Resultados: El ATO representó el 73,6\% de los casos hospitalizados por enfermedad inflamatoria pélvica, con frecuencia de 1,5 casos por mes y $17,2 \%$ de actinomicosis. La edad media de las pacientes fue 40,5 años. El dispositivo intrauterino (DIU) se asoció con ATO en $84,4 \%$ de los casos, $94,4 \%$ sin control y con media de uso de 10,2 años. El ATO unilateral fue el más frecuente $(57,8 \%)$ y la anexectomía unilateral la operación más común. El $17,2 \%$ de las pacientes presentaron complicaciones y el $85,9 \%$ quedaron con infertilidad. El costo total de los 64 casos fue \$86.331.713 (UF 3.788), con una media de \$1.348.933 (UF 59,2). Conclusión: Existe un aumento de la frecuencia del ATO y de la actinomicosis pélvica, con incremento consiguiente de la infertilidad y de los costos, asociados al uso de DIU, sin control y por tiempo prolongado.

\section{PALABRAS CLAVE: Absceso tuboovárico, dispositivo intrauterino, actinomicosis}

\section{SUMMARY}

Objective: To determine frequency and risk factors of tuboovarian abscess (TOA) and observe complications, fertility damages and surgical costs of medical-surgical treatment. Method: Retrospective study in 64 patients operated with TOA diagnosis. Patients were managed with medical-surgical treatment: use of antibiotics and then surgery in cases of peritonitis diffuse, persistent fever with palpable mass and adnexial mass greater than $6 \mathrm{~cm}$ without fever. They were evaluated in future fertility and complications: infection and of surgical wound dehiscence, reoperation by residual TOA, intestinal injury, bladder injury and medical complications. Results: The TOA accounted for $73.6 \%$ of hospitalized cases of pelvic inflammatory disease (PID), 1.5 cases per month and $17.2 \%$ of actinomycosis. The mean age of patients was 40.5 years. The intrauterine device (IUD) was associated with TOA in $84.4 \%$ of cases, $94.4 \%$ uncontrolled and with a 10.2 years mean use. The unilateral TOA was the most frequent $(57.8 \%)$ and the unilateral anexectomy the most common operation. The $17.2 \%$ of patients presented complications and $85.9 \%$ remained infertile. These results showed an increase compared with those obtained in the series published in 1993. The total cost of the 64 cases was $\$ 86.331 .713$ (UF 3.788), with a mean of $\$ 1.348 .933$ (UF 59.2). Conclusion: There is an 
increased frequency of the TOA and of pelvic actinomycosis, with consequent increase of infertility and costs associated with the uncontrolled and long-term use of IUD.

\section{KEY WORDS: Tuboovarian abscess, intrauterine device, actinomycosis}

\section{INTRODUCCIÓN}

El absceso tuboovárico (ATO) es una salpingitis severa que produce grandes daños en el aparato reproductor de la mujer (1). Su manejo requiere hospitalización prolongada y costosa. Las complicaciones luego del tratamiento quirúrgico (absceso de herida operatoria, reoperación, lesión intestinal, infección pleuropulmonar, sepsis, lesión vesical) son frecuentes y pueden ser causa de muerte de la paciente $(1,2)$. La obstrucción tubaria secundaria al daño anatómico causado por la infección e inflamación y principalmente la extirpación de los anexos enfermos, son causa usual de infertilidad en el ATO $(1,2)$.

Los factores de riesgo conocidos son: episodios de enfermedad inflamatoria pélvica (EIP) a repetición, historia de infertilidad, procedimientos intrauterinos (raspado biópsico, histerosalpingografía) y en Chile, el uso prolongado y sin control del dispositivo intrauterino (DIU) $(1,2)$.

En la literatura extranjera, sin embargo, la asociación EIP y DIU se menciona con una frecuencia muy baja ( $1 \%$ a $1 \%$ mujeres año uso) (3). Se afirma que el riesgo es durante el primer mes posterior a su inserción y existiría asociación con salpingitis leves y moderadas y no con ATO (4). Este bajo riesgo se incrementaría con el aumento de parejas sexuales y por la existencia de infecciones de transmisión sexual (ITS) durante el uso del DIU (5). Posterior al mes de uso son escasas las publicaciones que relacionan DIU con EIP.

La frecuencia del ATO ha disminuido en las publicaciones internacionales (6). En Chile, sin embargo, la incidencia de 39\% (1), mayor que en otros países, ha aumentado especialmente en mujeres mayores de 40 años $(7,8)$, a expensa de los casos asociados con el uso del DIU y principalmente producidos por Actinomyces (9). Estos microorganismos originan abscesos pélvicos múltiples y de gran severidad, con importantes adherencias que dificultan el diagnóstico y el manejo médico quirúrgico. Exige una cirugía laboriosa con alta posibilidad de daño intestinal y tratamiento antibiótico por tiempo prolongado.

EI DIU en nuestro país es insertado y manejado en los consultorios de Atención Primaria de acuerdo a normas que consideran criterios anticonceptivos, principalmente de duración del efecto anticonceptivo, desconociéndose los riesgos de infección intrauterina por el uso prolongado de este método. Es necesario normar el tiempo de uso del DIU con el objeto de disminuir la frecuencia del ATO, las complicaciones y el daño sobre la fertilidad de la mujer.

Los objetivos de este estudio fueron conocer la frecuencia y los factores de riesgo del ATO, en pacientes hospitalizadas en un establecimiento público de salud y observar las complicaciones, daños en la fertilidad y los costos del tratamiento médico quirúrgico.

\section{MATERIAL Y MÉTODO}

Se estudiaron retrospectivamente las pacientes operadas con el diagnóstico de ATO, en el Servicio de Obstetricia y Ginecología del Hospital Clínico San Borja Arriarán, durante el período comprendido entre el $1^{\circ}$ de enero de 2004 y el 31 de julio de 2007.

Se investigó el factor de riesgo asociado al ATO. Cuando existió DIU se extrajo durante el tratamiento antibiótico. En los DIU con cobre $(\mathrm{Cu})$ se describió el estado del $\mathrm{Cu}$. Se definió ATO como el hallazgo quirúrgico en trompa, ovario y estructuras vecinas comprometidas con inflamación, pus y adherencias, que alteran la anatomía del aparato genital superior $(10,11)$. El diagnóstico fue ratificado por el estudio anátomo patológico. Habitualmente el ATO se presenta luego de una inflamación crónica previa (salpingitis crónica), con oclusión del ostium tubárico. Posteriormente aparece exudado inflamatorio que se extiende hasta el ovario, con la formación de un ATO, que lleva a la obliteración de las relaciones anatómicas pélvicas. Su rotura desencadena un cuadro de peritonitis localizada o generalizada $(12,13)$.

Se definió actinomicosis como el compromiso anexial con formación de abscesos, fístulas y tejido fibroso denso que, en la mayoría de los casos, se presentó como "pelvis congelada" o pelvis sellada, por la incapacidad de liberar las estructuras comprometidas durante la cirugía. El diagnóstico morfológico se ratificó con la demostración de gránulos de azufre en el pus o en secciones histológicas de 
la muestra quirúrgica. Los gránulos representan grupos de bacilos Gram positivos ramificados. En las biopsias los filamentos se tiñen bien con Gomori-Grocott y con hematoxilina-eosina, pero se necesita de tinción especial (Gram plata) para mostrar que los gránulos están compuestos de bacterias en ramificación $(14,15)$.

Las pacientes fueron manejadas con criterio médico quirúrgico. Inicialmente con administración de antibióticos en esquema triasociado (penicilina G sódica 5 millones cada $6 \mathrm{~h}$ IV, metronidazol 500 mg cada 8 h IV y gentamicina 160 mg IV) y luego cirugía. En las mujeres con el antecedente de alergia a la penicilina se usó el esquema clindamicina-gentamicina. Los criterios de laparotomía fueron: a) peritonitis difusa; b) fiebre persistente con masa palpable luego de tres días de antibióticos y c) masa anexial mayor de $6 \mathrm{~cm}$ luego de mejoría clínica (6). Sin embargo algunas pacientes con el diagnóstico de pelviperitonitis, por decisión del médico residente tratante, fueron operadas de inmediato con una dosis de antibióticos. La colpoceliotomía se realizó con absceso pélvico abordable. En las pacientes con sospecha quirúrgica de actinomicosis ("pelvis congelada") se realizó exploración y de acuerdo a las posibilidades quirúrgicas, drenaje de absceso y mínima liberación de adherencias.

Durante el postoperatorio se mantuvo el mismo tratamiento antibiótico triasociado. El alta se dio entre las 72 a 96 horas de la operación y se indicó ciprofloxacino oral $500 \mathrm{mg}$ cada $12 \mathrm{~h}$ y metronidazol oral $500 \mathrm{mg}$ cada $8 \mathrm{~h}$ hasta completar 12-14 días en total de tratamiento. Las mujeres con actinomicosis se trataron al alta con doxiciclina $200 \mathrm{mg}$ por día durante tres a seis meses hasta la desaparición completa de la tumoración pélvica demostrada por clínica o por ultrasonografía.

Las pacientes fueron evaluadas en relación a complicaciones y secuelas:

Complicaciones: infección y dehiscencia de herida operatoria, reoperación por ATO residual, lesión intestinal, lesión vesical y complicaciones médicas. Se clasificaron en leves (infección y dehiscencia de herida operatoria, enterocolitis) y graves o severas (restantes complicaciones).

Secuelas: infertilidad, secundaria a la extirpación de los anexos enfermos durante la cirugía.

El cálculo del costo de manejo de los casos de ATO incluyó horas médicas, hospitalización en sala y en $\mathrm{UCl}$, exámenes de laboratorio, estudio anátomopatológico, ultrasonografía, antibióticos, otros medicamentos, curaciones e intervenciones quirúrgicas. Estos valores fueron proporcionados por las Unidades de Valorización y Departamento de Abastecimiento del Hospital Clínico San Borja Arriarán, el 31 de octubre de 2007. Se utilizó el valor de la UF de ese día: \$19.391,96 (US dólar promedio octubre 2007: $\$ 501,4)$.

El análisis estadístico se efectuó con la prueba de T de Student y Chi cuadrado según correspondiera. Se consideró como significativo un valor $p<0,05$.

\section{RESULTADOS}

Datos generales. Durante los 43 meses del estudio se operaron 64 pacientes con ATO. Representaron el 73,6\% (64/87) de los casos hospitalizados por EIP y con una frecuencia de 1,5 casos por mes. En la Tabla I se describe las características de la población.

Tabla I

CARACTERÍSTICAS GENERALES DE LA POBLACIÓN EN ESTUDIO

\begin{tabular}{ll}
\hline Variable & $\mathrm{n}=64$ \\
\hline Edad (años) & \\
$\quad$ Media \pm DE (rango) & $40,5 \pm 7,6(17-55)$
\end{tabular}

Paridad

Media \pm DE (rango) $\quad 2,3 \pm 1,1(0-6)$

ATO frecuencia mensual $\quad 1,5$

Actinomicosis n (\%)

$11(17,2)$

Hospitalización (días)

Media \pm DE (rango)

$14,9 \pm 13,4(6-86)$

Uso de antibióticos (días)

Mediana (rango)

$16(10-180)$

El $84,4 \%(54 / 64)$ de las mujeres eran usuarias de DIU al momento de la hospitalización (51 TCu, 1 Multiload y 2 Lippes) con una media de uso del mismo DIU de 10,2 \pm 4,7 años (rango: 2-24 años). En el 94,4\% (51/54) de los casos el DIU estaba sin control por más de 3 años. Se encontró pérdida total o casi total del $\mathrm{Cu}$ en 48/52 de los DIU con $\mathrm{Cu}$ (Tabla II).

El 17,2\% (11/64) de la pacientes presentó actinomicosis, asociada a DIU en todos los casos. El 
uso de antibióticos en ellas fue significativamente $(\mathrm{p}<0,00001)$ más prolongado $(78,6 \pm 49$ días $)$ que en los ATO sin actinomicosis (17,5 $\pm 8,3$ días).

\section{Tabla II \\ FACTORES ASOCIADOS}

\begin{tabular}{lcc}
\hline Factor & $\mathrm{n}$ & $\%$ \\
\hline Uso de DIU* & 54 & 84,4 \\
Ligadura tubaria & 6 & 9,4 \\
Infertilidad secundaria & 3 & 4,7 \\
EIP previa & 1 & 1,6 \\
\hline
\end{tabular}

DIU: dispositivo intrauterino. EIP: enfermedad inflamatoria pélvica. *Años de uso: media 10,2 \pm 4,7 (rango: 2-24 años)

Cirugía. El ATO unilateral fue el hallazgo intraoperatorio más frecuente $57,8 \%(37 / 64)$ y la anexectomía unilateral la operación más común (Tablas III y IV). Dos mujeres tuvieron más de dos operaciones. La primera paciente tuvo seis intervenciones quirúrgicas: en la primera operación se hizo anexectomía y salpingectomía contralateral y liberación de adherencias intestinales; en la segunda por absceso de la pared e intraabdominal, rotura intestinal, sepsis y SDR, se hizo drenaje de los abscesos, sutura de intestino delgado y laparostomía contenida; en la tercera operación se realizó aseo quirúrgico;

\section{Tabla III} DIAGNÓSTICO INTRAOPERATORIO

\begin{tabular}{lcc}
\hline Modalidad & $\mathrm{n}$ & $\%$ \\
\hline ATO unilateral & 37 & 57,8 \\
ATO bilateral & 19 & 29,7 \\
Pelvis congelada & 5 & 7,8 \\
Absceso pélvico & 3 & 4,7 \\
\hline
\end{tabular}

ATO: absceso tuboovárico.

en la cuarta cierre de laparostomía contenida; en la quinta y sexta operaciones se realizaron injertos en escaras de decúbito producidas durante sus hospitalizaciones. La segunda paciente tuvo tres laparotomías: en la primera fue drenado el ATO en el Hospital de Urgencia Asistencia Pública, luego en el Hospital Clínico San Borja Arriarán se hizo la segunda con histerectomía subtotal, anexectomía unilateral, resección y anastomosis de sigmoides distal y resección del íleon terminal e ileostomía; en la tercera operación se hizo cierre de la ileostomía. La cirugía se hizo en la mayoría de los casos $81,3 \%$ $(52 / 64)$ post tratamiento médico antibiótico y en las restantes $18,8 \%$ (12/64), se hizo inmediatamente por evidencias de peritonitis y compromiso del estado general.

Complicaciones y secuelas. Se presentan en la Tabla V. El 17,2\% (11/64) de las pacientes presentaron complicaciones: $41,7 \%(5 / 12)$ en aquellas

Tabla IV

TIPO DE OPERACIÓN

\begin{tabular}{lcc}
\hline Operación & $\mathrm{n}$ & $\%$ \\
\hline Anexectomía unilateral & 28 & 43,8 \\
Anexectomía bilateral & 10 & 15,6 \\
Anexectomía + salpingectomía contralateral & 9 & 14,1 \\
Laparotomía exploradora & 5 & 7,8 \\
Salpingectomía uni-bilateral & 5 & 7,8 \\
Histerectomía + anexectomía & 4 & 6,2 \\
Drenaje absceso pélvico + anexectomía & 3 & 4,7 \\
o salpingectomía uni o bilateral & &
\end{tabular}


que se realizó cirugía inmediata y 11,5\% (6/52) en las que recibieron tratamiento antibiótico previo, diferencia significativa $(p<0,05)$. Cuatro mujeres presentaron dos o más complicaciones. Ocho tuvieron complicaciones severas (reoperación por ATO, lesión intestinal, lesión vesical, SDR, plastrón abdominal, sepsis) y tres presentaron complicaciones leves (dos infección de herida operatoria, dehiscencia y resutura posterior y una con enterocolitis y deshidratación, probablemente secundaria al tratamiento antibiótico). La paciente con insuficiencia tubular aguda (recuperada totalmente) correspondió a un caso en que se usó Gentamicina por 30 días antes del ingreso al Servicio de Ginecología. Las complicaciones intestinales se presentaron en las dos pacientes con más de dos operaciones. El $85,9 \%(55 / 64)$ de las mujeres resultaron con infertilidad por la extirpación quirúrgica de los anexos enfermos.

Costos. El costo total de los 64 casos fue $\$ 86.331 .713$ (UF 3.788), con una media de $\$ 1.348 .933$ (UF 59,2) (Tabla VI).

\section{DISCUSIÓN}

Este estudio muestra que hubo un aumento de la frecuencia del ATO y de la actinomicosis pélvica, con incremento consiguiente de la infertilidad y de los costos, asociados al uso de DIU sin control y por tiempo prolongado.

Al comparar variables epidemiológicas y resul-

\section{Tabla V \\ COMPLICACIONES DEL TRATAMIENTO}

\begin{tabular}{lc}
\hline Complicación & $\mathrm{n}$ \\
\hline $\begin{array}{l}\text { Infección herida operatoria, } \\
\text { dehiscencia - resutura }\end{array}$ & 4 \\
$\begin{array}{l}\text { Síndrome dificultad respiratoria } \\
\text { Reoperación por absceso - } \\
\text { hematoma infectado }\end{array}$ & 2 \\
$\begin{array}{l}\text { Lesión intestinal } \\
\text { Escara Injerto }\end{array}$ & 2 \\
Lesión vesical & 2 \\
$\begin{array}{l}\text { Plastrón abdominal, } \\
\text { tratamiento médico }\end{array}$ & 1 \\
Sepsis & 1 \\
Enterocolitis - deshidratación herida operatoria, & 1 \\
\hline
\end{tabular}

Tabla VI COSTOS DEL TRATAMIENTO DEL ABSCESO TUVOOVÁRICO

\begin{tabular}{lcccc}
\hline & \multicolumn{2}{c}{ Costo unitario (media) } & \multicolumn{2}{c}{ Costo total de casos } \\
& $\$$ & $U^{*}$ & $\$$ & UF \\
\hline Hospitalización & 955.462 & 49,271 & 61.149 .567 & $3.153,346$ \\
UCl & 121.757 & 6,279 & 7.792 .477 & 401,841 \\
Exámenes, ecografías & 23.379 & 1,206 & 1.496 .250 & 77,158 \\
Antibióticos & 76.848 & 3,963 & 4.918 .257 & 253,624 \\
Biopsias & 10.494 & 0,541 & 671.640 & 34,635 \\
Operaciones & 160.993 & 8,299 & 10.303 .522 & 531,330 \\
Total ${ }^{\star *}$ & 1.348 .933 & 69,559 & 86.331 .713 & $4.451,934$ \\
\hline
\end{tabular}

UCI: Unidad de cuidado intensivo. UF: Unidad de fomento. *Valor UF \$19.391,9 al 31/10/07. **Rango \$ 665.649 (UF 34,3) - \$11.410.283 (UF 588,4) 
tados clínicos entre esta serie de ATO y la serie publicada en 1993 (1) por este autor, con casos hospitalizados en este Servicio, se observó que la frecuencia del ATO en el Hospital Clínico San Borja Arriarán ha aumentado. El ATO representó el 74\% de las mujeres hospitalizadas por EIP aguda durante el período en estudio ( $39 \%$ en 1993), con aumento de la frecuencia de 0,6 a 1,5 casos mensuales. Paralelamente aumentó la frecuencia de ATO por Actinomyces (10\% a $17,2 \%)$ modalidad de mayor gravedad, por su difícil manejo médico quirúrgico y por la mayor posibilidad de complicaciones. Como consecuencia de lo anterior se produjo un incremento de la infertilidad (75\% a $86 \%$ ) por la extirpación de los anexos enfermos y finalmente, por el manejo de estas pacientes hubo un aumento de los gastos en el Servicio de Ginecología.

Este aumento de la frecuencia del ATO también se menciona en otras publicaciones. En California (6) en un estudio que comparó dos períodos (1991 y 2001) a pesar que la tasa de hospitalización por EIP y por ATO decreció respectivamente desde 2,6 a 1,0 y desde 0,6 a 0,4 por 10.000 mujeres, hubo un aumento de la hospitalización por ATO de 24 a $39 \%$ en las mayores de 40 años. No obstante, no se mencionan los factores de riesgo asociados, $\mathrm{ni}$ el uso de DIU. Del mismo modo, en Noruega, comparando dos períodos (1990-1992 y 2000-2002) a pesar de la reducción en 35\% de las EIP hospitalizadas, se encontró un incremento significativo de los casos de ATO (26 a 43\%). Tampoco se mencionan los factores de riesgo asociados (16).

El aumento de la frecuencia de ATO encontrada en este trabajo (duplica al encontrado en 1993) podría atribuirse a la mayor asociación ATO/DIU (84\% de los casos vs $49 \%$ en 1993), con mayor tiempo de uso del mismo DIU (10,2 años de media) y a la ausencia de control (94\%) del mismo.

En trabajos extranjeros, sin embargo, se menciona una frecuencia muy baja de asociación entre EIP y DIU (1\% a $1 \%$ mujeres año uso), en series de casos de TCu $(3,17)$. En dos revisiones sistemáticas sobre relación entre EIP y DIU, con 45 y 365 artículos estudiados respectivamente $(3,4)$, la EIP se presenta como salpingitis leve, durante el primer mes post inserción (3-5) y en pacientes con ITS concomitante $(3-5,17)$. No se describe la asociación con ATO (4). Tampoco en estas revisiones se mencionan los factores de riesgo, ni el tiempo de uso del mismo DIU. Sin embargo, en otras publicaciones recientes, el DIU (TCu) se asocia con salpingitis severa, en mujeres mayores de 35 años de edad (7) y en pacientes que van a cirugía por ATO, de edad mayor (media 45 años) y uso prolongado de DIU (6,8 años) (8).
En este estudio el incremento del ATO asociado a DIU, TCu, sin control y uso mayor de 10 años, en mujeres mayores de 40 años, se puede explicar por los siguientes argumentos:

1) Aumento de la colonización bacteriana en superficie del DIU con mayor tiempo de uso. Se sabe que con uso prolongado del DIU (especialmente mayor de 5 años) el número de UF colonias $\left(10^{6}\right.$ y $10^{7}$ ó más) y el número de especies bacterianas por paciente (hasta 6 especies asociadas) aumenta significativamente en el bíofilm bacteriano que se forma en la superficie del DIU (18). Esta asociación microbiana es preferentemente a expensas de bacterias aerobias y anaerobias Gram positivas. La presencia de bacterias endógenas y la asociación microbiana favorecen el desarrollo de salpingitis severa (1).

2) Capacidad antibacteriana del Cu y relación con tiempo de uso del DIU. Con el uso prolongado es posible la desaparición del $\mathrm{Cu}$ y de su efecto. Actualmente es conocida la actividad antibacteriana que tiene el $\mathrm{Cu}(19,20)$ y otros metales sobre algunas bacterias Gram positivas, Gram negativas y hongos. En esta serie se encontró pérdida total o casi total del $\mathrm{Cu}$ en casi todos los DIU extraídos durante la hospitalización. Es posible que el $\mathrm{Cu}$ impidiera la aparición del ATO antes de los 10 años de uso. Así puede entenderse que en la serie del año 93, (con usuarias de dispositivos $\sin \mathrm{Cu}$, Lippes u otros, en más de la mitad de los casos) el ATO apareció a los 6,7 años de uso del DIU. Existiría coIonización e infección bacteriana del dispositivo sin $\mathrm{Cu}$ con menor tiempo de uso. Este concepto debe ser demostrado en el futuro.

3) Mayor susceptibilidad a las infecciones pelvianas a mayor edad de la mujer. Se sabe en la actualidad que la mayor edad de la paciente, constituye un factor de riesgo en la producción del ATO $(7,8)$. Es posible que esta condición sea secundaria al tiempo mayor de uso del DIU. Pero también es probable que con el aumento de la edad disminuya la microbiota vaginal normal representada por lactobacilos, favoreciéndose el ascenso microbiano al interior del útero. Los bajos niveles de estrógenos en la perimenopausia, que podrían tener estas pacientes, pueden participar en la disminución de la flora lactobacilar y favorecer el desarrollo de infecciones. Esto indudablemente debe ser demostrado.

El manejo y tratamiento de las 64 pacientes con ATO fue de $\$ 86.331 .713$ (UF 3.788); este gasto equivale al costo de 416 partos vaginales atendidos en el Hospital Clínico San Borja Arriarán (costo unitario de cada parto vaginal \$207.378).

En relación con los resultados de este estudio es conveniente seguir las siguientes recomendaciones en el uso del DIU con $\mathrm{Cu}$, para evitar o dis- 
minuir la frecuencia del ATO, sus complicaciones, el daño sobre la fertilidad de la mujer y los gastos producidos en el manejo de esta patología:

a) la usuaria de DIU debe controlarse por matrona 1 vez al año a lo menos y por médico cada vez que exista infección cérvico vaginal (ICV), la que siempre debe tratarse;

b) la mujer que ha tenido ICV a repetición, debe ser evaluada por médico entre los 3 y 5 años de uso del DIU, para decidir extracción o continuidad del uso;

c) recambio del DIU a los 5 años de uso en las mujeres que han tenido ICV o infecciones urinarias recurrentes o mayores de 40 años;

d) extracción del DIU en todas las mujeres en menopausia.

\section{CONCLUSIÓN}

El uso de DIU, sin control y por tiempo prolongado se asocia a un aumento de la frecuencia del ATO, de la actinomicosis pélvica, de la infertilidad y de los costos. Se efectúan recomendaciones que enfatizan el control anual de las usuarias, el tratamiento de la infección cérvico vaginal, la extracción del DIU a los 5 años en mujeres mayores de 40 años y en menopausia.

\section{BIBLIOGRAFÍA}

1. Ovalle A, Martínez MA, Casals A, Yuhaniak R, Giglio MS. Estudio clinico y microbiológico de la enfermedad inflamatoria pélvica aguda. Rev Chil Obstet Ginecol 1993;58:103-12.

2. Ovalle A, Roncone S, Bayo F, Herrera R, Schafer C, Pérez $M$, et al. Bacteriología y fundamentos del tratamiento médico quirúrgico del absceso tuboovárico. Rev Chil Obstet Ginecol 1985;50:257-72.

3. Campbell SJ, Cropsey KL, Matthews CA. Intrauterine device use in a high-risk population: experience from an urban university clinic. Am J Obstet Gynecol 2007;197:193.e1-6.

4. Meirik O. Intrauterine devices - upper and lower genital tract infections. Contraception 2007;75(6 Suppl): S41-7

5. Mohllajee AP, Curtis KM, Peterson HB. Does insertion and use of an intrauterine device increase the risk of pelvic inflammatory disease among women with sexually transmitted infection? A systematic review. Contraception 2006;73:145-53.
6. Paik CK, MD, Waetjen LE, Xing G, Dai J, Sweet RL. Hospitalizations for pelvic inflammatory disease and tuboovarian abscess. Obstet Gynecol 2006;107:6116.

7. Viberga I, Odlind V, Berglund L. Older age is a risk factor for pelvic inflammatory disease in intrauterine device users. Acta Obstet Gynecol Scand 2005; 4: 202-7.

8. Halperin R, Levinson O, Yaron M, Bukovsky I, Schneider D. Tubo-ovarian abscess in older women: is the woman's age a risk factor for failed response to conservative treatment? Gynecol Obstet Invest 2003;55:211-5.

9. Wolff M, Levy I, Ballesteros J. Actinomicosis: experiencia con ocho casos. Rev Méd Chile 1988;116:11724.

10. Monif G. Clinical staging of acute bacterial salpingitis and its therapeutic ramifications. Am J Obstat Gynecol 1982;143:489-95.

11. Jacobson L. Differential diagnosis of acute pelvic inflammatory disease. Am J Obstet Gynecol 1980;138:1006-11.

12. Rosai J. Rosai and Ackerman's Surgical Pathology. Ninth edition. 2004.

13. Kurman J. Blaustein's Pathology of the female genital tract. Fifth edition. 2002

14. Alegría J, González M, Galleguillos M, Whittle C, Franco C. Revisión de infección pelviana por Actinomices: presentación de un caso clínico. Rev Chil Radiol 2003;9(4):196-200.

15. Madrid F, Díaz S, Mucientes F, Klaassen R. Actinomicosis Ginecológica. Rev Chil Obstet Ginecol 2003;68(1):21-7.

16. Sørbye IK, Jerve F, Staff AC. Reduction in hospitalized women with pelvic inflammatory disease in Oslo over the past decade. Acta Obstet Gynecol Scand 2005;84:290-6.

17. Stanback J, Shelton JD. Pelvic inflammatory disease attributable to the IUD: modeling risk in West Africa. Contraception 2008;77:227-9.

18. Pál Z, Urbán E, Dósa E, Pál A, Nagy E. Biofilm formation on intrauterine devices in relation to duration of use. J Med Microbiol 2005;54:1199-203.

19. El-Behery M, El-Twigry H. Synthesis, magnetic, spectral, and antimicrobial studies of $\mathrm{Cu}(\mathrm{II}), \mathrm{Ni}(\mathrm{II}) \mathrm{Co}(\mathrm{II})$, $\mathrm{Fe}$ (III), and UO2(II) complexes of a new Schiff base hydrazone derived from 7-chloro-4-hydrazinoquinoline. Spectrochim Acta A Mol Biomol Spectrosc 2007;66:2836.

20. Gudasi KB, Patil MS, Vadavi RS. Synthesis, characterization of copper(II), cobalt(II), nickel(II), zinc(II) and cadmium(II) complexes of [7-hydroxy-4-methyl-8-coumarinyl] glycine and a comparitive study of their microbial activities. Eur J Med Chem 2008;43(11):2436-41. 